Article

\title{
Analysis of Dynamic Characteristics and Power Losses of High Speed on/off Valve with Pre-Existing Control Algorithm
}

\author{
Qi Zhong ${ }^{1,2,3, *}$, Xiele Wang ${ }^{1,3}$, Geng Xie ${ }^{1,3}$, Huayong Yang ${ }^{2}$, Cheng $\mathrm{Yu}^{1,3}$, Enguang $\mathrm{Xu}^{1,3}$ and Yanbiao $\mathrm{Li}^{1,3, *}$ \\ 1 College of Mechanical Engineering, Zhejiang University of Technology, Hangzhou 310023, China; \\ 2111902258@zjut.edu.cn (X.W.); 2111902259@zjut.edu.cn (G.X.); 2112002427@zjut.edu.cn (C.Y.); \\ 2111902022@zjut.edu.cn (E.X.) \\ 2 The State Key Laboratory of Fluid Power and Mechatronic Systems, Zhejiang University, \\ Hangzhou 310027, China; yhy@zju.edu.cn \\ 3 The Key Laboratory of Special Purpose Equipment and Advanced Processing Technology, \\ Ministry of Education and Zhejiang Province, Zhejiang University of Technology, Hangzhou 310023, China \\ * Correspondence: zhongqi@zjut.edu.cn (Q.Z.); lybrory@zjut.edu.cn (Y.L.)
}

check for updates

Citation: Zhong, Q.; Wang, X.; Xie, G.; Yang, H.; Yu, C.; Xu, E.; Li, Y. Analysis of Dynamic Characteristics and Power Losses of High Speed on/off Valve with Pre-Existing Control Algorithm. Energies 2021, 14, 4901. https://doi.org/10.3390/ en14164901

Academic Editors: Mario Marchesoni and Nicu Bizon

Received: 12 July 2021

Accepted: 9 August 2021

Published: 11 August 2021

Publisher's Note: MDPI stays neutral with regard to jurisdictional claims in published maps and institutional affiliations.

Copyright: (c) 2021 by the authors. Licensee MDPI, Basel, Switzerland. This article is an open access article distributed under the terms and conditions of the Creative Commons Attribution (CC BY) license (https:/ / creativecommons.org/licenses/by/ $4.0 /)$.

\begin{abstract}
A high-speed on/off valve (HSV) is generally the core component of a digital hydraulic transmission system. Therefore, its dynamic characteristics often restrict the overall performance of the digital hydraulic system. Most of the current studies focus on the optimization on the dynamic characteristics or the energy characteristics, few studies have comprehensively considered the two characteristics of the valve together. In this paper, a pre-existing control algorithm (PECA) is proposed to improve the dynamic characteristics of the HSV, and simultaneously optimize the power losses of the HSV to improve its energy conversion efficiency. The results show that, compared with the traditional single-voltage driven strategy, the opening time of the PECA decreases by $29.4 \%$, the closing time decreases by $59.6 \%$, and the energy conversion rate increases by $7.9 \%$.
\end{abstract}

Keywords: power loss; solenoid valve; dynamic response; temperature rising; multiphysics methodology

\section{Introduction}

A HSV is widely used in hydraulic transmission control systems because of its fast response, small size and strong antipollution ability, especially in high frequency hydraulic control systems [1-3]. The response speed and control accuracy of the HSV control system is largely determined by the dynamic response characteristics of the HSV itself $[4,5]$. On the other hand, with the successive reduction of traditional energy and the serious problems of environmental pollution and global warming, the energy-saving problem of hydraulic systems needs to be solved urgently [6-8]. As a basic component of hydraulic transmission controlling system, the energy loss of the HSV affects the energy efficiency of the whole system. Therefore, the dynamic characteristics and energy characteristics are the two important performance indexes of the HSV. It is of great significance to optimize two performances simultaneously.

At present, there are many studies focused on improving the dynamic characteristics of the HSV by optimizing the driving strategy. Li et al. [9] proposed a double voltage driving strategy to a generate larger driving current and smaller maintenance current, which effectively improved the response characteristics of the HSV. On this basis, multivoltage source circuits $[10,11]$ have been studied, which can shorten the closing time of the HSV by generating reverse voltage. Zhong et al. [12] proposed the PWM (pulse width modulation) control method, which used current feedback to achieve adaptive adjustment of the duty cycle, and finally improved the working frequency of the HSV. In addition, Zhong et al. [13] proposed an adaptive oil pressure method that can make the driving current change adaptively according to the oil pressure, and then reducing the switching time of the HSV. With the increase in the switching frequency of the HSV, its input power and energy 
loss also greatly increased. To explore the power loss law of the HSV, Tan et al. [14] analyzed the energy characteristics of the HSV at different operating frequencies, and their results indicated that with the increase in operating frequency (the working frequency of the HSV), the maximum temperature (the maximum temperature of the HSV in the working process) of the HSV increased, which greatly increased the power loss of the HSV. However, the above researches mainly focus on improving the dynamic characteristics and switching frequency of the HSV; the influence of the energy field and the thermal field on its performance is rarely considered.

The power losses of HSV are mainly divided into core loss, stranded loss and solid loss [15]. Many scholars have studied the influence of electrical parameters on power losses of HSV. Zhao et al. [16] studied the influence of different boosting on the dynamic characteristics and power losses of HSV: they found that an increase in the driving voltage is conducive to the rapid opening action of the HSV, but it would greatly increase the core loss. Cheng et al. [15] explored the influence of the driven voltage on the power loss of the HSV, which showed that the driving strategy is the key factor affecting the power losses of the solenoid injector. Zhao [17] studied the influence of different driving currents on the power losses of the HSV. With the decrease in driving current, the opening response characteristics of the HSV becomes worse, but the power loss ratio decreases, and the energy efficiency of the HSV is improved. These studies explored the relationship between the valve's electrical parameters and power losses, considering the dynamic characteristics in detail. However, they only gave the general trend of the dynamic characteristic, electrical parameters and energy losses, and did not focus on how to improve the dynamic characteristics of the HSV. The general law of dynamic characteristics-energy loss change cannot obtain the precise driving strategy to optimize the dynamic characteristics of the HSV.

Due to the fact that the power losses of the HSV are dissipated in the form of heat, it is difficult to accurately measure the power losses of the HSV in experimental settings. Therefore, it is necessary to build an accurate thermodynamic model of the HSV to effectively achieve thermal field analysis. In the prior art, the lumped parameter method [18,19], the semi-analytical method [20-22], and the numerical method [23] are often used for thermodynamic model design and analysis.

To sum up, in the above research, few strategies can both improve the dynamic characteristics of the HSV and also optimize its energy loss characteristics. Therefore, the contribution of this paper is to propose PECA to improve the dynamic characteristics of the HSV and its energy conversion efficiency. The purpose is to make HSV take into account the advantages of high performance and green energy saving. To optimize the dynamic characteristics and power losses of the HSV simultaneously, in this paper, a multi-physical field coupling model is established to analyze the coupling relationship between motion state, dynamic characteristics and power losses. The change rule of the dynamic characteristics and the power consumption loss of the HSV are obtained. The proposed algorithm is used to optimize the dynamic characteristics of the HSV, and at the same time make the HSV conserve energy, thereby making it environmentally friendly.

\section{The Mathematical Model of the HSV}

\subsection{Structure and Operating Principle of the HSV}

The researched HSV in this paper is a two-position two-way HSV, which adopts the structure of normal-closed internal flow poppet valve. Its main components are coil, valve core, valve body, return spring, end cover, as shown in Figure 1.

The switching action of the HSV is realized by an H-bridge circuit, as shown in Figure 2. When the rising edge of the control signal occurs, the $\mathrm{Q}_{1}$ and $\mathrm{Q}_{4}$ tubes are opened and the $\mathrm{Q}_{2}$ and $\mathrm{Q}_{3}$ tubes are closed; the HSV will be loaded by the positive voltage-it generates electromagnetic force to attract the valve core to the right, and the HSV is opened. Similarly, when the failing edge of the control signal occurs, the $Q_{2}$ and $Q_{3}$ are opened and the $Q_{1}$ and $\mathrm{Q}_{4}$ are closed; the HSV is loaded with negative voltage and the current of the coil will drop to 
0 rapidly, as will the electromagnetic force. When the coil is powered off, the return spring pushes the core valve to the left, and the HSV is closed.

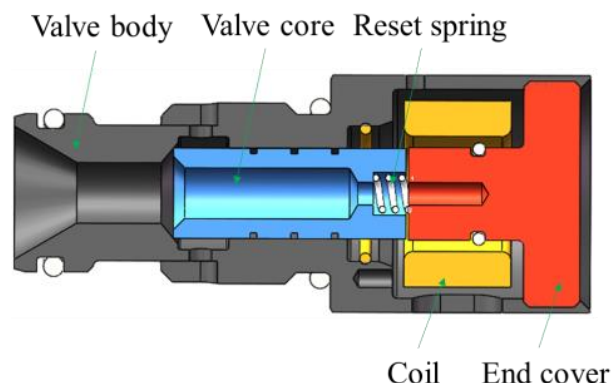

Figure 1. Structure of the HSV.

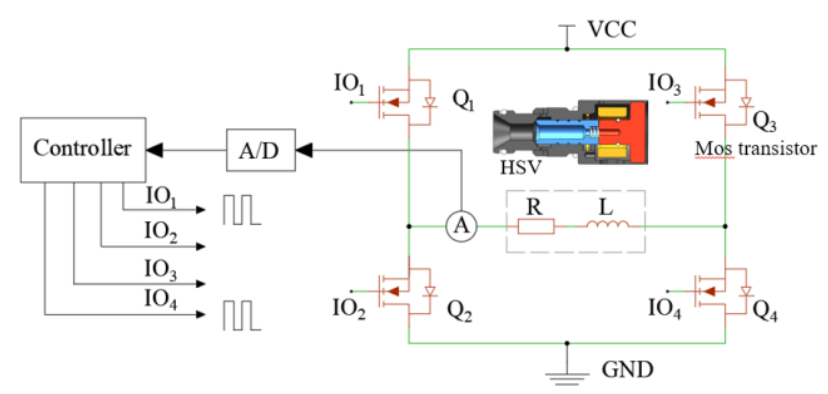

Figure 2. Equivalent circuit of the HSV.

\subsection{The Motion Characteristic Model of the HSV}

The HSV is affected by the mechanical field, the electromagnetic field and the thermal field [24]. To analyze its performance effectively, the key is to establish an accurate multiphysical field coupling model. Each physical model is derived as follows.

When the valve core of the HSV moves, it is mainly affected by electromagnetic force $F_{\mathrm{m}}$, hydraulic force $P_{\mathrm{s}} A$, spring return force $F_{\mathrm{r}}$, steady flow force $F_{\mathrm{s}}$, transient flow force $F_{\mathrm{t}}$ and damping force $F_{\mathrm{v}}$. The transient motion equation of the spool is obtained:

$$
m \frac{\mathrm{d}^{2} x}{\mathrm{~d} t^{2}}=F_{\mathrm{m}}-\left(F_{\mathrm{t}}+F_{\mathrm{s}}\right)-F_{\mathrm{r}}-c \frac{\mathrm{d} x}{\mathrm{~d} t}-P_{\mathrm{s}} A
$$

where $m$ is the mass of the moving parts of the HSV, $x$ is the displacement of the valve core, $t$ is the time, $c$ is the damping coefficient, $P_{\mathrm{s}}$ is the pressure of the oil inlet, and $A$ is the effective cross-sectional area of the oil inlet.

The damping force can be expressed as:

$$
F_{v}=c \frac{d x}{d t}
$$

Considering the magnetic field parameters, the electromagnetic force of the HSV can be expressed as:

$$
F_{\mathrm{m}}=\frac{\phi^{2}}{2 \mu_{0} S}
$$

where $\mu_{0}$ is the air permeability, $\phi$ is the magnetic flux, $S$ is the section area of the air gap.

Flow force is one of the main axial forces acting on the valve core, which is composed of steady flow force and transient flow force. The steady-state hydrodynamic force can be expressed as:

$$
F_{\mathrm{s}}=P_{\mathrm{s}} A-\rho q_{v} \cdot\left(v_{2} \cos \alpha-v_{1}\right)
$$

where $\rho$ is the fluid density, $q_{v}$ is the flow rate of the HSV, $v_{2}$ is the oil flow rate at the outlet port, and $v_{1}$ is the oil flow rate at the inlet port, $\alpha$ is the half cone angle of the valve seat. 
The transient flow force can be expressed as [25]:

$$
F_{\mathrm{t}}=C_{\mathrm{d}} w L_{\mathrm{d}} \sqrt{2 \rho \Delta P} \frac{\mathrm{d} x}{\mathrm{~d} t}
$$

where $C_{\mathrm{d}}$ is the flow coefficient, $w$ is the area gradient, $L_{\mathrm{d}}$ is the damping length, $\rho$ is the density of oil, and $\Delta P$ is the pressure difference through the orifice.

Without considering the influence of the temperature on the resistance, the dynamic equation of the coil circuit of the HSV can be expressed as follows:

$$
U=I R+L \frac{\mathrm{d} I}{\mathrm{~d} t}=I R+\frac{\mathrm{d} \psi}{\mathrm{d} t}
$$

The relationship between inductance and magnetic flux is as follows:

$$
L=\frac{\mathrm{d} \psi}{\mathrm{d} t}=N \frac{\mathrm{d} \phi}{\mathrm{d} t}
$$

Therefore, the coil excitation voltage can be expressed as:

$$
U=I R+L \frac{\mathrm{d} I}{\mathrm{~d} t}=I R+N \frac{\mathrm{d} \phi}{\mathrm{d} t}
$$

where $U$ is the excitation voltage of the coil, $I$ is the coil current, $R$ is the coil resistance, $\psi$ is the coil flux, $L$ is the coil inductance, $N$ is the number of coil turns.

\subsection{The Energy and Temperature Field Model of the HSV}

In the working process of the HSV, the coil is electrified to produce a magnetic field, which produces an electromagnetic force to attract the valve core to move. In this process, a large amount of energy is lost and finally dissipated in the form of heat, and only a small portion of the energy is converted into kinetic energy. The power loss of the HSV mainly includes core loss, stranded loss and solid loss.

The power loss caused by materials during current conduction is called stranded loss, and the material resistivity is the cause of it. Stranded loss is also known as the Joule Lenz effect, and it can be expressed as follows [26]:

$$
P_{\mathrm{s}}=\frac{1}{\sigma_{\mathrm{c}}} \int_{\text {vol }} J^{2} \mathrm{~d} V_{\mathrm{w}}
$$

where $J$ is the current density, $\sigma_{\mathrm{c}}$ (Siemens/s) is the conductivity of copper and $V$ is the winding volume.

When the HSV works, an eddy current will be generated in the valve core and cause the core loss. Bertotti [27] separated core loss into three parts: hysteresis loss $P_{\mathrm{h}}$, classical eddy current loss $P_{\mathrm{c}}$ and excess loss $P_{\mathrm{e}}$. According to Bertotti's energy loss theory, the loss of these three parts can be expressed by the following three formulas [15]:

$$
\begin{aligned}
& P_{\mathrm{h}}=k_{\mathrm{h}} f B_{\mathrm{m}}^{2} \\
& P_{\mathrm{c}}=k_{\mathrm{c}} f B_{\mathrm{m}}^{2} \\
& P_{\mathrm{e}}=k_{\mathrm{e}} f B_{\mathrm{m}}^{2}
\end{aligned}
$$

where, $k_{\mathrm{h}}$ is the hysteresis loss coefficient, $k_{\mathrm{c}}$ is the classical eddy current loss coefficient, $k_{\mathrm{e}}$ is the excess loss coefficient, $f$ is the operating frequency of the driving signal, and $B_{\mathrm{m}}$ is the amplitude of magnetic induction.

Therefore, the total core loss can be expressed as:

$$
P_{\mathrm{i}}=P_{\mathrm{h}}+P_{\mathrm{c}}+P_{\mathrm{e}}=k_{\mathrm{h}} f B_{\mathrm{m}}^{2}+k_{c} f^{2} B_{\mathrm{m}}^{2}+k_{\mathrm{e}} f^{1.5} B_{\mathrm{m}}^{1.5}
$$


Solid loss represents the loss of bulk soft magnetic materials due to induced current [26]:

$$
P_{\mathrm{s}}=\frac{1}{\sigma_{\mathrm{s}}} \int_{\text {vol }} J^{2} \mathrm{~d} V_{s}
$$

where $\sigma_{\mathrm{s}}$ is the conductivity of bulk soft magnetic materials and $V$ is the volume of bulk soft magnetic material.

All these losses will eventually release to the outside in the form of heat. Part of the heat will be transferred along the radial direction and finally radiate to the outside by convection between the body shell and the air. The other part of the heat will be transferred along the axial direction of the valve core, which will transfer to the positioning bolt and finally radiated to the outside by convection.

According to the equivalent heat circuit method [28], the heat flow in the HSV can be equivalent to the vector combination of axial $x$ and radial $r$. Therefore, heat transfer models are established for the two directions.

The axial heat transfer equation, initial conditions and boundary conditions can be expressed by the following formula:

$$
\left\{\begin{array}{l}
\frac{d^{2} T}{d x^{2}}=-\frac{q_{\mathrm{v}}}{\lambda} \\
\left.T\right|_{t=0}=T_{0} \\
-\lambda S \frac{d T}{d x}=q_{0},\left.T\right|_{x=L}=T_{L}
\end{array}\right.
$$

where $T$ is the temperature, $x$ is the axial coordinate point, $q_{\mathrm{v}}$ is the heat source, $\lambda$ is the thermal conductivity, $S$ is the effective cross-sectional area of heat transfer, $q_{0}$ is the heat flow in the HSV.

The radial heat transfer equation, initial conditions and boundary conditions can be expressed by the following formula:

$$
\left\{\begin{array}{l}
\frac{d T}{d r}=-\frac{1}{2 \lambda} q_{\mathrm{v}} r \\
\left.T\right|_{r=0}=T_{0} \\
\frac{q_{\mathrm{v}} r_{0}}{2}=\alpha\left(T_{0}-T_{x}\right),\left.T\right|_{r=r_{0}}=T_{L}
\end{array}\right.
$$

where $r$ is the radial coordinate point, $\alpha$ is the thermal conductivity of the heat transfer surface, $T_{0}$ is the temperature of the heat dispersing surface, and $T_{x}$ is the medium temperature around the heat dispersing surface.

\section{Simulation Results and Analysis}

Using Maxwell and workbench, the mechanical field, magnetic field, temperature field and other multi field coupling analysis of the HSV are carried out.

\subsection{Simulation Analysis of Dynamic Characteristics of the HSV}

Figure 3 shows the PECA and conventional single-voltage driven strategy. The traditional single-voltage driven strategy uses a $24 \mathrm{~V}$ voltage square wave to drive the HSV, and PECA is divided into five stages, and a multivoltage phase strategy is used to drive the HSV.

Figure 4 shows the dynamic characteristics of the HSV excited by the traditional single-voltage driven strategy at the operating frequency of $20 \mathrm{~Hz}$. In these conditions, the opening delay time of the HSV is $0.6 \mathrm{~ms}$, the opening moving time of the HSV is $1.1 \mathrm{~ms}$, closing delay time of the HSV is $4.6 \mathrm{~ms}$, the closing moving time of the HSV is 2.4 ms. Figure 5 shows the dynamic characteristics of the HSV excited by PECA at the operating frequency of $20 \mathrm{~Hz}$. At this point, the opening delay time of the HSV is $0.1 \mathrm{~ms}$, the opening moving time of the HSV is $1.1 \mathrm{~ms}$, the closing delay time of the HSV is $0.5 \mathrm{~ms}$, and the closing moving time is $1.4 \mathrm{~ms}$. Compared with the traditional single-voltage driven strategy, PECA can reduce the opening time (consisting of the opening moving time and the opening delay time) of the HSV by $29.4 \%$ and the closing time (consists of the closing 
moving time and the closing delay time) of the HSV by $72.6 \%$, greatly improving the dynamic characteristics of the HSV. Table 1 records the dynamic characteristics of the HSV under the two strategies.

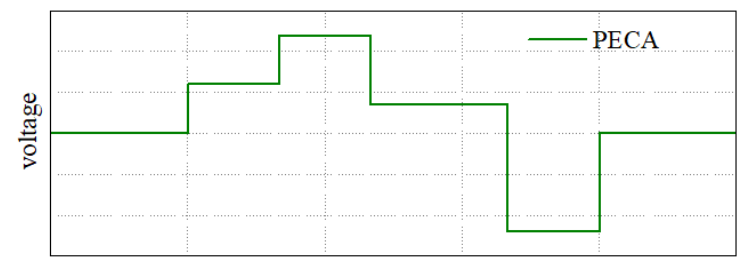

time

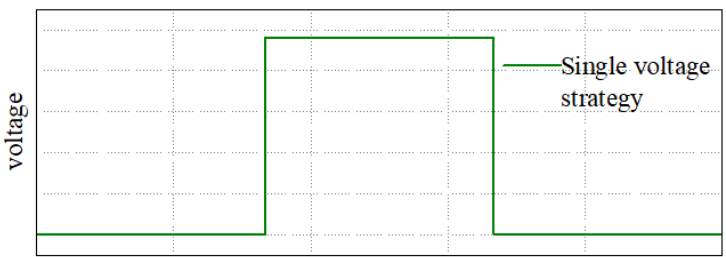

Figure 3. The two driven strategies.

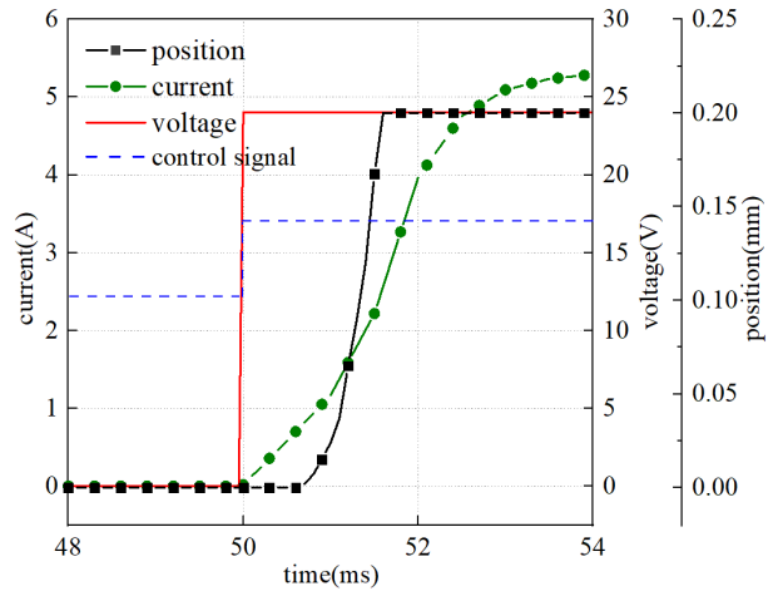

(a)

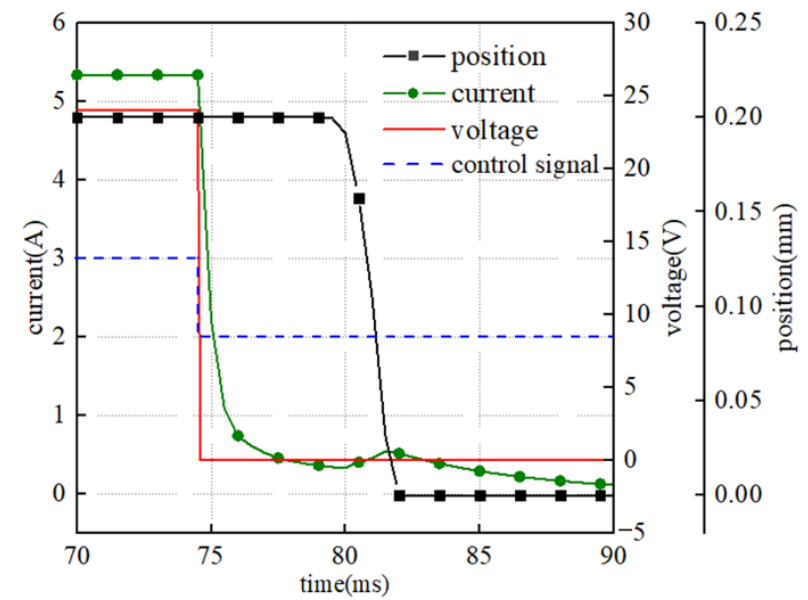

(b)

Figure 4. The dynamic characteristics' curve in single voltage strategy: (a) Opening process; (b) Closing process.

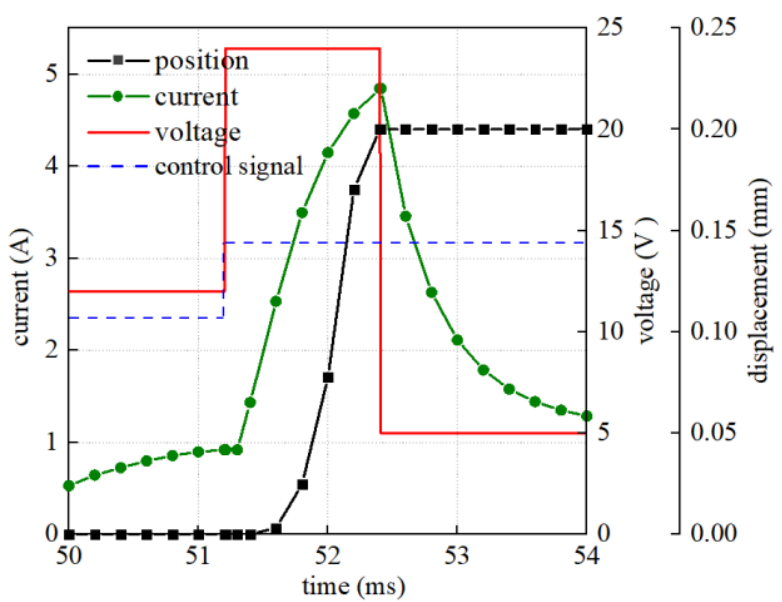

(a)

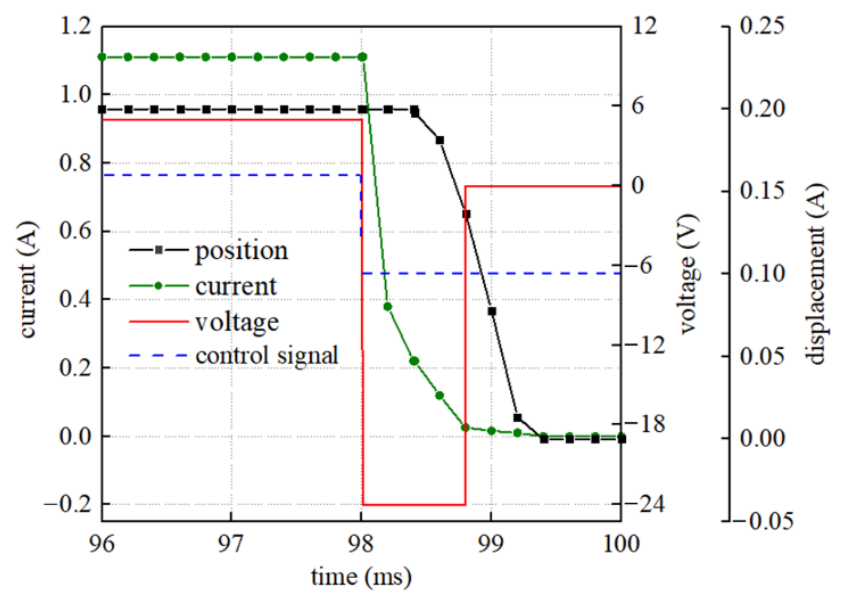

(b)

Figure 5. The dynamic characteristics' curve in PECA: (a) Opening process; (b) Closing process. 
Table 1. Dynamic characteristics of the HSV in the two driven strategy.

\begin{tabular}{ccc}
\hline & Single Voltage & PECA \\
\hline ODT (ms) & 0.6 & 0.1 \\
OMT (ms) & 1.1 & 1.1 \\
CDT (ms) & 4.6 & 0.5 \\
CMT (ms) & 2.4 & 1.4 \\
\hline
\end{tabular}

For clarity, the opening delay time, opening moving time, are simplified to ODT, OMT, respectively, and the closing delay time, closing moving time are simplified to CDT, CMT, respectively.

Under the same simulation conditions, the amplitude-frequency characteristics of the traditional single-voltage driven strategy and PECA are analyzed, and the phase-frequency characteristics of the HSV in the closing phase under the two algorithms are analyzed. The simulation results are shown in Figure 6.

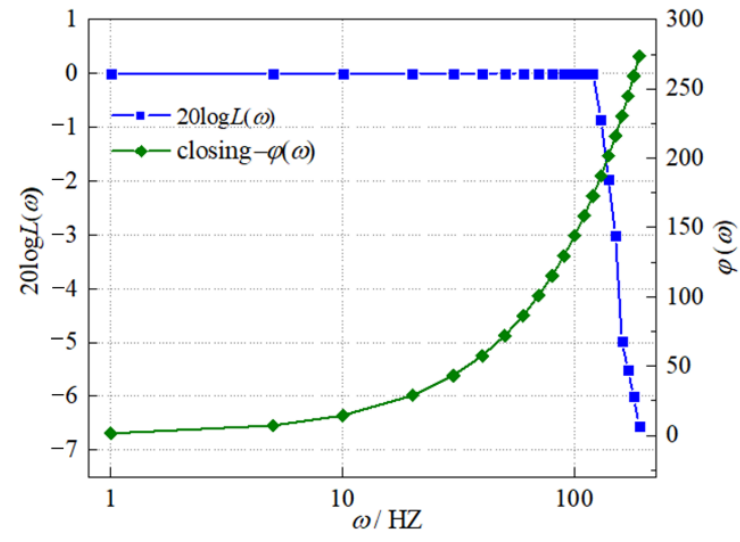

(a)

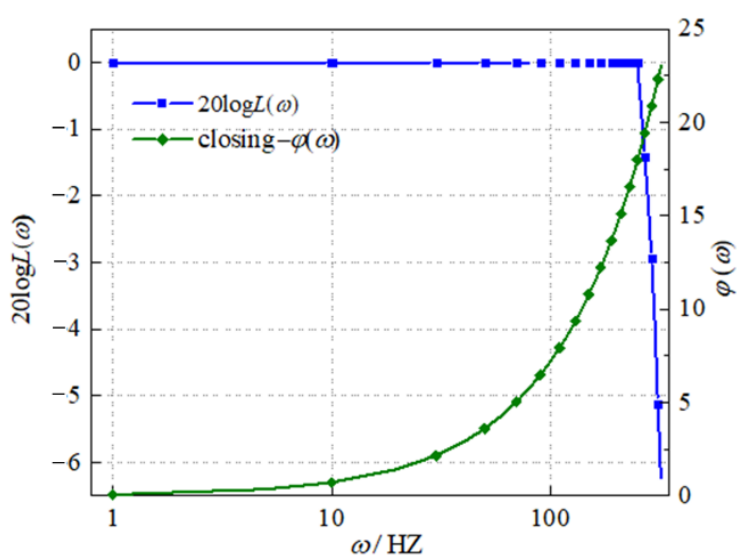

(b)

Figure 6. Frequency characteristics in the two driven strategies: (a) Amplitude-frequency curve in single voltage strategy; (b) Amplitude-frequency curve in PECA.

As can be seen from Figure 6a, under the traditional single-voltage driven strategy, when the duty ratio is $50 \%$, the maximum operating frequency that can achieve the whole switching action is $120 \mathrm{~Hz}$. The operating frequency corresponding to the $90^{\circ}$ lagging phase angle in the closing phase is $65 \mathrm{~Hz}$. When the operating frequency is less than $65 \mathrm{~Hz}$, the HSV can achieve the whole switching action. When the operating frequency is $65 \mathrm{~Hz} 120 \mathrm{~Hz}$, although the HSV can complete the switching action, the lagging phase angle is greater than $90^{\circ}$, which will reduce the real-time performance of the control system. When the frequency is greater than $120 \mathrm{~Hz}$, the HSV cannot complete the switching action.

As can be seen from Figure 6 b, under the PECA, when the duty ratio is $50 \%$, the maximum operating frequency that can achieve the whole switching action is $250 \mathrm{~Hz}$. At the same time, the operating frequency corresponding to the $90^{\circ}$ lagging phase angle in the closing phase is more than $250 \mathrm{~Hz}$, so, the lagging phase angle cannot be used as a condition to limit the movement of the HSV. When the operating frequency is greater than $250 \mathrm{~Hz}$, the valve cannot complete the switching action. Compared with the traditional single-voltage control strategy, the range of the controllable frequency is increased by $108 \%$.

\subsection{Simulation Analysis of Energy Characteristics of the HSV}

To describe the power loss of the HSV more accurately, the transient three-dimensional field simulation analysis of the HSV is carried out. Figure 7 shows the power loss curves of the HSV under two strategies. Although the loss curve in Figure $7 \mathrm{a}$ is different from that in Figure $7 \mathrm{~b}$, the change trend of each loss in the two figures is consistent. It can be found that the trend of the stranded loss is same as current. Core loss and solid loss mainly occur in the opening and closing phases, and are related to the change rate of the current. 


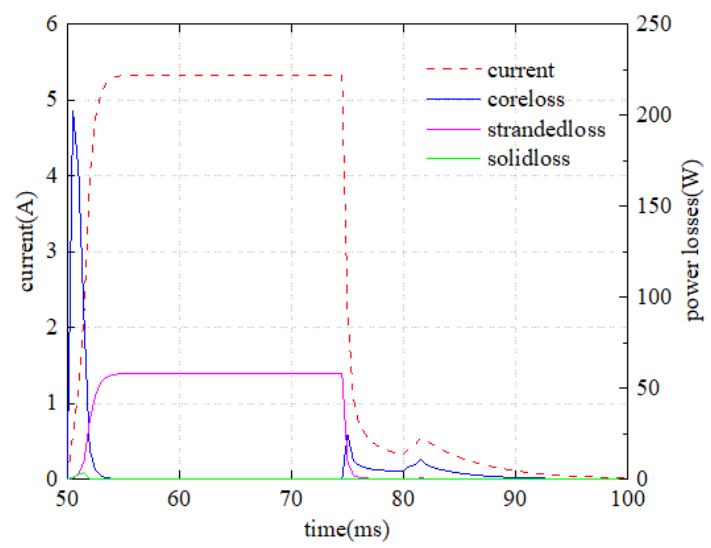

(a)

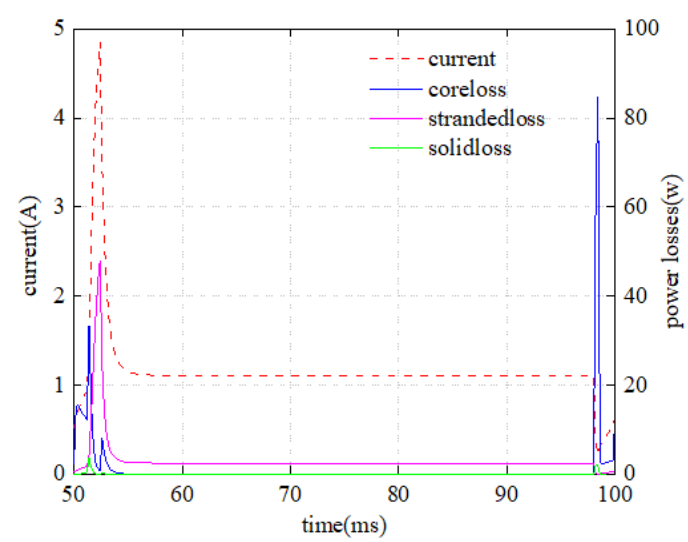

(b)

Figure 7. Power loss curve in the two driven strategies: (a) Power loss curve in single voltage strategies; (b) Power loss curve in PECA.

Figure 6 shows that under the traditional single-voltage driven strategy, the HSV can complete a switching action within the operating frequency at $0 \sim 65 \mathrm{~Hz}$, while under PECA, the range of operating frequency is $0 \sim 250 \mathrm{~Hz}$, this determines the range of the operating frequency in the simulation. Figure 8 shows the energy ratio of the HSV at different operating frequencies under the traditional single-voltage driven strategy. It can be seen form Figure 8 that stranded loss accounts for the largest proportion, followed by core loss, while solid loss and mechanical energy are relatively low. With the increase in the operating frequency, the stranded loss ratio gradually decreases, while the other three kinds of energy ratio gradually increase. It is worth noting that under the traditional single-voltage strategy, the energy conversion efficiency (mechanical energy ratio) of the HSV is very low.

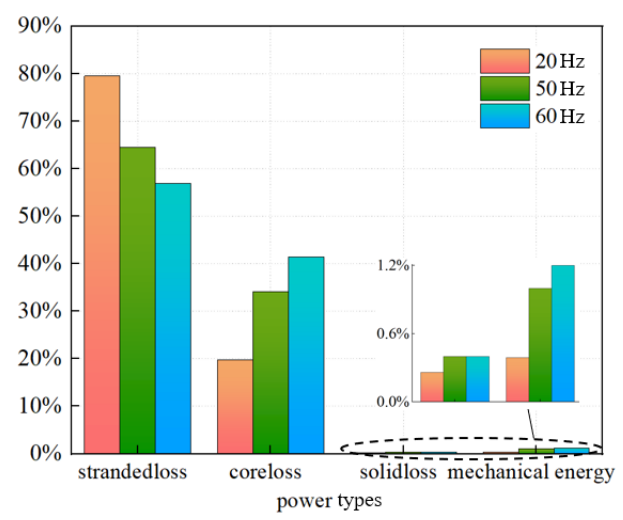

Figure 8. Power proportion in single voltage strategy.

As the operating frequency rises from $20 \mathrm{~Hz}$ to $60 \mathrm{~Hz}$, the mechanical energy ratio increases from $0.4 \%$ to $1.2 \%$. A large amount of input power is dissipated in the form of energy loss.

As can be seen from Figure 7a, under the traditional single-voltage driven strategy, the current is maintained at the maximum value for a long time, which generates a large amount of stranded loss. Increasing the operating frequency can effectively reduce the time when the current is maintained at a high value and reduce stranded loss. However, under the traditional single-voltage strategy, the range of the operating frequency of the HSV is narrow $(0 \sim 65 \mathrm{~Hz})$. The operating frequency of the HSV limits the effect of energy consumption optimization.

The PECA is proposed to optimize the defects of the power loss and the low energy consumption ratio of the HSV. Figure 9 shows the current curves for both the two strategies. The peak current and average current under PECA are less than those under conventional 
single-voltage driven strategy. This reduces the total input power of the HSV and reduces the power loss of the valve.

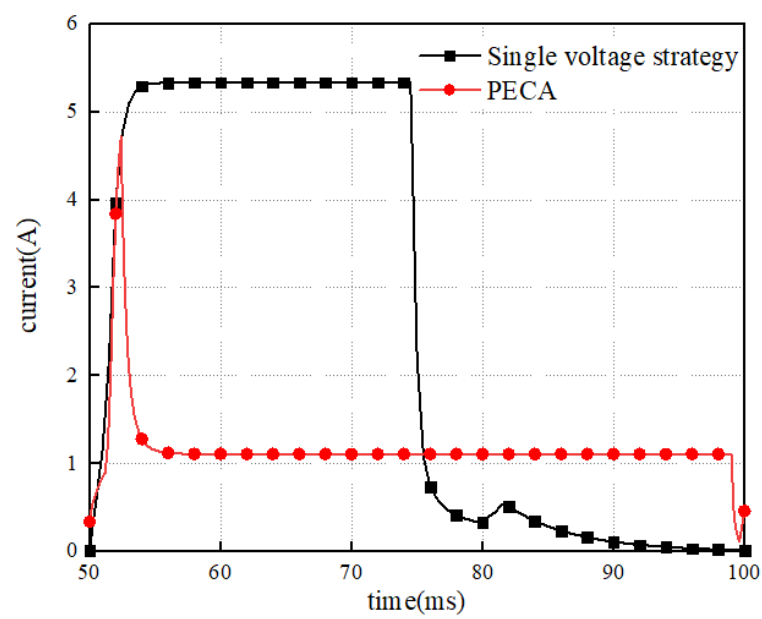

Figure 9. The current curve in two driven strategies.

Under the PECA excitation, the power losses ratio of the HSV at different operating frequencies are shown in Figure 10. With the increase in the operating frequency, the stranded loss gradually decreases, while the other energy ratios increase. Stranded loss declined by $22.2 \%$ while core loss increases by $13.8 \%$. In addition, solid loss accounts for a low proportion and increases by $3.5 \%$. The proportion of mechanical efficiency was improved, increasing from $3.0 \%$ to $7.9 \%$. The results show that the operating frequency has a great influence on stranded loss and core loss, but the influence on solid loss is not obvious. Moreover, the PECA can improve the energy conversion rate of the HSV, compared with the traditional single-voltage driven strategy, the maximum energy conversion rate increases from $1.2 \%$ to $7.9 \%$.

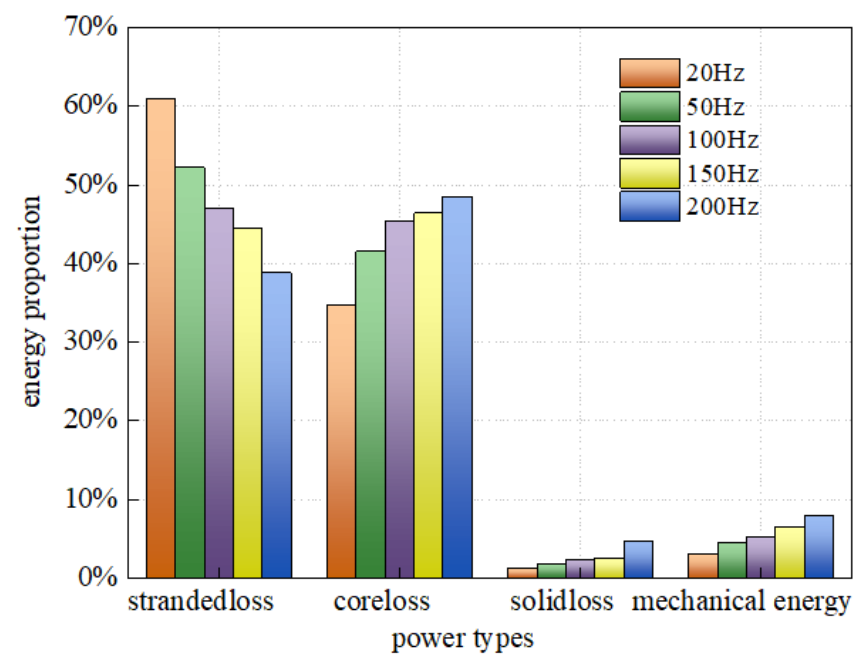

Figure 10. Power proportion in PECA.

According to the simulation results above, the PECA can effectively improve the dynamic characteristics of the HSV and expand the range of the operating frequency of the HSV. At the same time, PECA can effectively improve the energy loss of the HSV, greatly reduce the stranded loss, and improve the energy conversion efficiency.

\section{Experimental Results and Analysis}

Due to the power losses dissipating into the air in the form of heat, the value of power losses directly measured in experiment is not accurate enough to be used for analysis. As 
the power losses of the HSV will be eventually converted into heat energy and cause the temperature of the valve body to rise, the temperature rise can indirectly measure the power loss of the HSV [13]. In this paper, the temperature measurement method is used to indirectly measure power loss, and to verify the ability of PECA to optimize the power losses of the HSV.

Figure 11 shows the temperature rise diagram of the HSV under the two driven strategies. The results show the valve temperature is stabilized at $121^{\circ} \mathrm{C}$ at $18 \mathrm{~min}$ under the traditional single-voltage driven strategy, while the valve temperature stabilized at $62{ }^{\circ} \mathrm{C}$ at 8 min under the PECA, with a $48 \%$ drop in temperature, which means a lower value of the power loss. In addition, under PECA excitation, the slope of the temperature rise curve of the HSV is lower, which indicates that the power losses of the HSV are smaller in unit time. Moreover, PECA enables the HSV to reach a stable working state in a shorter time.

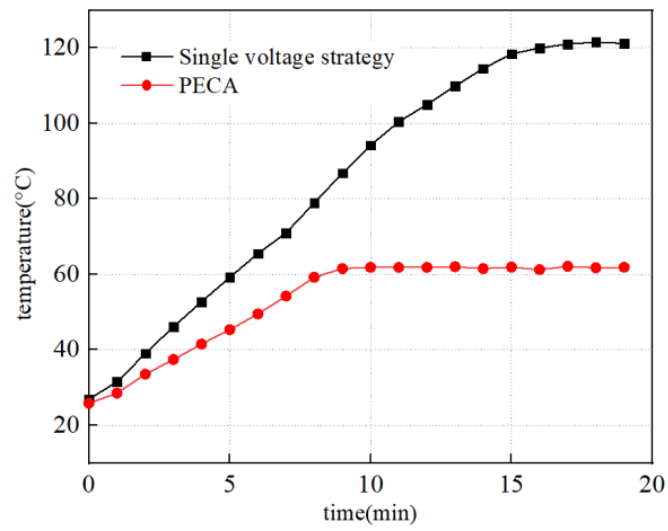

Figure 11. The temperature rise curve in two driven strategies.

Figure 12 shows the temperature of the HSV rises significantly at the coil area. When the HSV starts to operate, the temperature of the coil area is the highest, and with the increase in time, the temperature of other parts also increases gradually, but the coil still has the highest temperature area. This shows that the stranded loss is the main reason for the temperature rise of the HSV. According to Figure 9, the PECA effectively reduces the peak current and average current, so the stranded loss is greatly reduced, and then the maximum temperature increases.

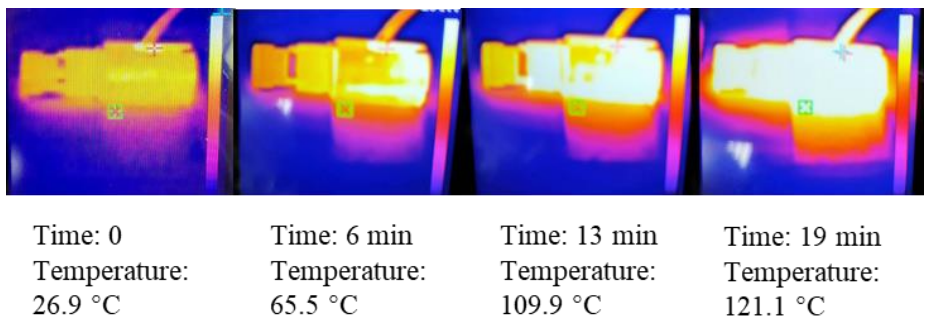

(a)

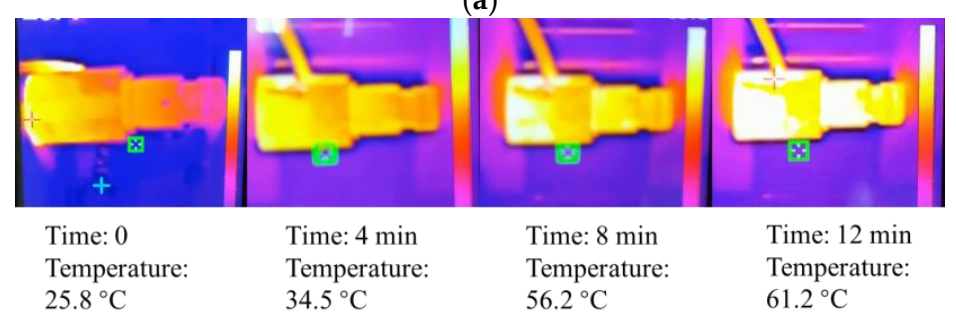

(b)

Figure 12. The temperature nephogram in two driven strategies: (a) The temperature nephogram in single voltage strategy at $20 \mathrm{~Hz}$; (b) The temperature nephogram in PECA at $20 \mathrm{~Hz}$. 
In conclusion, the experimental results show that PECA can effectively optimize the power losses of the HSV, and verify the effectiveness of energy simulation.

\section{Conclusions}

Dynamic characteristics and energy characteristics are two important performance indicators of the HSV. With the aggravation of energy and environmental problems, research on energy loss of the HSV is becoming more and more important. In the existing studies, there are few driving strategies that can optimize two performance indicators simultaneously. In this paper, the PECA is proposed to simulate and experimentally verify the dynamic characteristics and power losses of the HSV, and finally optimize the two performances indexes simultaneously.

The main conclusions are as follows:

(1) The PECA proposed in this paper can effectively improve the dynamic characteristics of the HSV, reducing the opening time by $29.4 \%$ and closing time by $59.6 \%$. In addition, the PECA extends the range of the controllable frequency of the HSV by $108 \%$.

(2) The stranded loss ratio is the largest in the HSV. The stranded loss will decrease with the increase in the operating frequency, while the core loss, solid loss and mechanical energy will increase.

(3) The PECA can significantly increase the energy conversion rate of the HSV from $1.2 \%$ to $7.9 \%$.

Core loss is the main portion of the power losses of the HSV, which is affected by many factors. The change law of core loss was not considered deeply in this study. In future studies, we will explore the influence of the frequency, temperature, material properties and other factors on core loss, and further improve the energy efficiency of the HSV.

Author Contributions: Conceptualization, Q.Z. and X.W.; methodology, Q.Z. and X.W.; software, G.X.; validation, H.Y.; formal analysis, X.W. and Q.Z.; investigation, E.X.; resources, Q.Z.; data curation, G.X., C.Y. and E.X.; writing—original draft preparation, X.W.; writing—review and editing, X.W.; visualization, Y.L. and H.Y.; supervision, Y.L.; project administration, X.W.; funding acquisition, Q.Z. All authors have read and agreed to the published version of the manuscript.

Funding: This research was funded by Open Foundation of the State Key Laboratory of Fluid Power and Mechatronic Systems, grant GZKF-201906, National Natural Science Foundation of China, grant \#52005441, Natural Science Foundation of Zhejiang province, grant LQ21E050017 and China Postdoctoral Science Foundation, grants \#2021M692777 and \#2021T140594.

Institutional Review Board Statement: Not applicable.

Informed Consent Statement: Not applicable.

Data Availability Statement: The data presented in this study are available on request from the corresponding author.

Acknowledgments: We thank Ren Yan for her valuable comments and suggestions on this article.

Conflicts of Interest: The authors declare no conflict of interest.

\section{References}

1. Cheong, K.H.; Doihara, R.; Shimada, T.; Terao, Y. Gravimetric system using high-speed double switching valves for low liquid flow rates. Meas. Sci. Technol. 2018, 29, 75304-75317. [CrossRef]

2. Jinlong, D.; Jiashun, S.; Cong, L.; Tianbiao, Y. Research of Pneumatic Polishing Force Control System Based on High Speed On/off with PWM Controlling. Robot. Comput. Integr. Manuf. 2021, 70, 102133-102146.

3. Jiao, Z.X.; Zhang, H.; Shang, Y.X.; Liu, X.C.; Wu, S. A power-by-wire aircraft brake system based on high-speed on-off valves. Aerosp. Sci. Technol. 2020, 106, 106177-106186. [CrossRef]

4. Qiu, H.Y.; Su, Q. Simulation Research of Hydraulic Stepper Drive Technology Based on High Speed On/Off Valves and Miniature Plunger Cylinders. Micromachines 2021, 12, 438. [CrossRef]

5. Shi, H.; Liu, Z.Y.; Wang, H.T. Design and performance analysis of hydraulic switching valve driven by magnetic shape memory alloy. Advances in Mechanical Engineering 2021, 13, 1-15. [CrossRef] 
6. Mahato, A.C.; Ghoshal, S.K. Energy-saving strategies on power hydraulic system: An overview. Proc. Inst. Mech. Eng. Part I J. Syst. Control Eng. 2021, 235, 147-169. [CrossRef]

7. Yin, X.Q.; Feng, Z.J. Analysis and Research on Energy Saving of Hydraulic Power Unit. In Materials Science, Energy Technology and Power Engineering Iii; You, Z., Xiao, J., Tan, Z., Eds.; AIP Publishing LLC: Melville, NY, USA, 2019; Volume 2154.

8. Cai, S.N.; Jiao, Y.; Xu, C.T.; Liang, Y.; Qin, R. Energy-saving Driver Circuit of High-speed Solenoid Valve Based on Softswitch Technology. In Proceedings of the 2nd International Conference on Instrumentation and Measurement, Computer, Communication and Control (IMCCC), Harbin, China, 8-10 December 2012; pp. 598-601.

9. Li, P.X.; Su, M.; Zhang, D.B. Iop. Response characteristic of high-speed on/off valve with double voltage driving circuit. In Proceedings of the 2nd International Conference on Design, Materials, and Manufacturing, Beijing, China, 23-25 June 2017; Volume 220, pp. 1757-8981.

10. Lee, I.Y. Switching response improvement of a high speed On/Off solenoid valve by using a 3 power source type valve driving circuit. In Proceedings of the IEEE International Conference on Industrial Technology, Bombay, India, 15-17 December 2006; pp. 2179-2184.

11. Zhong, Q.; Xie, G.; Wang, X.L.; Li, Y.; Yang, H.; Zhang, B.; Chen, B. Performance Analysis of High Speed on/off Valve by Multi-voltages Compound Excitation. J. Mech. Eng. 2021, 57, 191-201.

12. Zhong, Q.; Zhang, B.; Yang, H.Y.; Ma, J.E.; Fung, R.F. Performance analysis of a high-speed on/off valve based on an intelligent pulse-width modulation control. Adv. Mech. Eng. 2017, 9, 1687814017733247. [CrossRef]

13. Zhong, Q.; He, X.J.; Li, Y.B.; Zang, B.; Yang, H.; Chen, B. Research on Control Algorithm for High-speed on/off Valves that Adaptive to Supply Pressure Changes. J. Mech. Eng. 2021, 57, 224-235.

14. Tan, C.; Li, B.; Ge, W.Q. Thermal Quantitative Analysis and Design Method of Bistable Permanent Magnet Actuators Based on Multiphysics Methodology. IEEE Trans. Ind. Electron. 2020, 67, 7727-7735. [CrossRef]

15. Cheng, Q.; Zhang, Z.D.; Xie, N.L. Power losses and dynamic response analysis of ultra-high speed solenoid injector within different driven strategies. Appl. Therm. Eng. 2015, 91, 611-621. [CrossRef]

16. Zhao, J.; Wang, M.; Wang, Z.; Grekhov, L.; Qiu, T.; Ma, X. Different boost voltage effects on the dynamic response and energy losses of high-speed solenoid valves. Appl. Therm. Eng. 2017, 123, 1494-1503. [CrossRef]

17. Zhao, J.H.; Yue, P.F.; Grekhov, L.; Ma, X.Z. Hold current effects on the power losses of high-speed solenoid valve for com-mon-rail injector. Appl. Therm. Eng. 2018, 128, 1579-1587. [CrossRef]

18. Pfister, P.D.; Perriard, Y. Very-high-speed slotless permanent-magnet motors: Analytical modeling, optimization, design, and torque measurement methods. IEEE Trans. Ind. Electron. 2010, 57, 296-303. [CrossRef]

19. Bracikowski, N.; Hecquet, M.; Brochet, P.; Shirinskii, S.V. Multiphysics modeling of a permanent magnet synchro-nous machine by using lumped models. IEEE Trans. Ind. Electron. 2012, 59, 2426-2437. [CrossRef]

20. Yi, W.; Dan, M.I.; Staton, D. Ultrafast steady-state multiphysics model for PM and synchronous reluctance ma-chines. IEEE Trans. Ind. Appl. 2015, 51, 3639-3646.

21. Grobler, A.J.; Holm, S.R.; Schoor, G.V. Empirical parameter identification for a hybrid thermal model of a high speed permanent magnet synchronous machin. IEEE Trans. Ind. Electron. 2018, 65, 1616-1625. [CrossRef]

22. Shi, H.; Gong, G.F.; Yang, H.Y.; Mei, X.S. Energy Saving Analysis of Segment Positioning in Shield Tunneling Machine. J. Cent. South Univ. 2014, 21, 4526-4536. [CrossRef]

23. Sun, X.; Cheng, M. Thermal analysis and cooling system design of dual mechanical port machine for wind power application. IEEE Trans. Ind. Electron. 2013, 60, 1724-1733. [CrossRef]

24. Liu, Y.F.; Mao, M.C.; Xu, X.Y.; Shi, G. Multi-physics coupled thermo-mechanics analysis of a hydraulic solenoid valve. In Proceedings of the International Conference on Mechatronics and Industrial Informatics (ICMII 2013), Guangtzhou, China, 23-24 March 2013; Volume 321-324, pp. 102-107.

25. Fang, W.M.; Cheng, L.L.; Fu, X.; Zhou, H.; Yang, H.Y. Investigation on steady-state flow force of spool valve with U-grooves. J Zhejiang Univ. (China) 2010, 44, 574-580.

26. Ansoft Maxwell 2019 User's Manual, Ver.19.0 Design Reference; ANSYS, Inc. ANSYS Europe: Canonsburg, PA, USA, 2017; Available online: https: / / ansyshelp.ansys.com (accessed on 10 June 2021).

27. Bertotti, G. General properties of power losses in soft ferromagnetic materials. IEEE Trans. Magn. 1988, 24, 621-630. [CrossRef]

28. Lombardi, L.; Raimondo, R.; Antonini, G. Electrothermal formulation of the partial element equivalent circuit method. Int. J. Numer. Model. Electron. Netw. Devices Fields 2018, 31, e2253. [CrossRef] 